\title{
Identification of the Bleeding Point in Hemorrhagic Moyamoya Disease Using Fusion Images of Susceptibility- Weighted Imaging and Time-of-Flight MRA
}

\author{
(D) A. Miyakoshi, (D). Funaki, (D). Fushimi, (D). Kikuchi, (D). Kataoka, (DK. Yoshida, (D). Mineharu, (D).C. Takahashi, and
}

(D) S. Miyamoto

\begin{abstract}
BACKGROUND AND PURPOSE: The location of intracerebral hemorrhage in Moyamoya disease is a prognostic factor for rebleeding and the degree of preventive effects obtainable with bypass surgery. We evaluated whether the bleeding point and responsible vessel were detectable using fusion images of SWI and time-of-flight MRA performed during chronic-phase hemorrhage.
\end{abstract}

MATERIALS AND METHODS: We retrospectively enrolled 42 patients with hemorrhagic Moyamoya disease (48 hemorrhagic events). Fusion images of SWI and MRA were made using workstations, and we defined the bleeding point as the point at which the signal of an abnormally extended artery on MRA overlapped the hypointense area on SWI. Two independent raters identified the bleeding point, and classified the location and responsible vessels.

RESULTS: The bleeding point was detectable at a frequency of $79.2 \%$ by rater 1 . Agreement for the presence of a bleeding point was high (interrater $\kappa=0.83 ; 95 \% \mathrm{Cl}, 0.65-1$; intrarater $\kappa=0.86 ; 95 \% \mathrm{Cl}, 0.68-1$ ). The frequency of a periventricular location of the bleeding point was $65.8 \%$ by rater 1 , and agreement on the location was again high (interrater $\kappa=0.92 ; 95 \% \mathrm{Cl}, 0.82-1$; intrarater $\kappa=0.85 ; 95 \% \mathrm{Cl}, 0.72-0.99)$. The choroidal artery was the most frequent responsible vessel $(57.9 \%$ by rater 1$)$, and agreement on the responsible vessel was high (interrater $\kappa=0.84$; $95 \% \mathrm{Cl}$, 0.69-1; intrarater $\kappa=0.90 ; 95 \% \mathrm{Cl}, 0.78-1$ ).

CONCLUSIONS: Detection of the bleeding point in hemorrhagic Moyamoya disease using SWI and MRA fusion images offers highly reproducible results.

ABBREVIATION: ICH = intracerebral hemorrhage

ntracerebral hemorrhage ( $\mathrm{ICH})$ is one of the major factors affecting prognosis for patients with Moyamoya disease. ${ }^{1}$ $\mathrm{ICH}$ associated with Moyamoya disease is more common in adult patients and is mostly located in the parenchyma and ventricle. ${ }^{2}$ Dilated perforating and choroidal arteries developing as part of the collateral network are supposed to represent 1 source of hemorrhage. ${ }^{3,4}$ Direct bypass surgery for hemorrhagic-onset Moyamoya disease has been reported to contribute to the prevention of recurrent bleeding. ${ }^{5-8}$ Furthermore, the hemorrhage location is associated with the incidence of rebleeding and the degree of preventative effect achievable with bypass surgery. ${ }^{9}$ Accurate identification of the bleeding

Received February 18, 2019; accepted after revision July 29.

From the Departments of Neurosurgery (A.M., T.F., T.K., H.K., K.Y., Y.M. S.M.) and Diagnostic Imaging and Nuclear Medicine (Y.F.), Kyoto University Graduate School of Medicine, Kyoto, Japan; and Department of Neurosurgery (J.C.T.), National Cerebral and Cardiovascular Center, Osaka, Japan.

Please address correspondence to Akinori Miyakoshi, MD, Department of Neurosurgery, Kyoto University Graduate School of Medicine, 54 Kawahara-cho, Shogoin, Sakyo-ku, Kyoto 606-8507, Japan; e-mail: myks-knr@umin.ac.jp http://dx.doi.org/10.3174/ajnr.A6207 point and the vessel responsible for hemorrhage is thus clinically important.

Although some reports have investigated the distribution of microbleeds in Moyamoya disease, ${ }^{10,11}$ no reports have addressed the bleeding point and vessels responsible for symptomatic hemorrhage related to Moyamoya disease, with the exception of 1 study using head CT. ${ }^{12}$ Determining the site from which bleeding has occurred on CT and MR imaging performed during acutephase hemorrhage is often difficult.

MRA performed using a $3 \mathrm{~T}$ scanner has proved useful for evaluating the abnormally extended collateral networks in Moyamoya disease. ${ }^{13}$ The present study evaluated the reproducibility of fusion images of SWI and TOF-MRA performed during the chronic phase of hemorrhage to detect the precise bleeding point. Furthermore, we created a distribution map of bleeding points and investigated the vessels responsible for hemorrhage.

\section{MATERIALS AND METHODS}

This cross-sectional study was approved by the ethics committee at Kyoto University Graduate School of Medicine. 


\section{Patients}

We retrospectively enrolled consecutive patients diagnosed with symptomatic hemorrhagic Moyamoya disease who visited Kyoto University Hospital between January 2009 and April 2018. The diagnosis of Moyamoya disease was determined according to the proposed criteria. ${ }^{14}$ Patients who had undergone emergent hematoma evacuation craniotomy during the acute stage of hemorrhage were excluded because the responsible vessel might have been blocked. Patients who presented with primary subarachnoid hemorrhage, had undergone specific treatments for peripheral aneurysms, or for whom SWI or TOF-MRA images were unavailable were excluded. Cases in which fusion images could not be constructed because of an error caused by image processing at scanning were also excluded.

\section{Imaging Protocol and Postimaging Processing}

A 3T MR scanner (Magnetom Trio; Skyra, Prisma; Siemens, Erlangen, Germany) using a 32-channel head coil, which successfully reveals abnormal collateral vessels in Moyamoya disease, was introduced to our institution in 2009. Imaging parameters for SWI and TOF-MRA in this study were as follows-SWI: TR, $28 \mathrm{~ms}$; TE, $20 \mathrm{~ms}$; flip angle, $15^{\circ}$; FOV, $230 \times 179 \mathrm{~mm}$; matrix, $320 \times 250$; section thickness, $1 \mathrm{~mm}$; 128 slices; axial acquisition; acceleration factor of 2 using generalized autocalibrating partially parallel acquisition; scan time, 4 minutes 53 seconds; TOF-MRA: TR, 20-21 ms; TE, $3.69 \mathrm{~ms}$; flip angle, 20; FOV, $220 \times$ $187 \mathrm{~mm}$; matrix, $384 \times 328$; section thickness, $0.7 \mathrm{~mm}$; generalized autocalibrating partially parallel acquisition of 3; scan time, 5 minutes 48 seconds. The imaging field extended from the level of the foramen magnum to beyond the upper margin of the body of the lateral ventricle. Since 2016, the imaging field has been extended to the top of the head.

With the use of SWI and TOF-MRA images scanned during the chronic phase of ICH ( $\geq 3$ weeks since onset), we generated SWI and TOF-MRA axial fusion images on a workstation (Aquarius iNtuition Viewer, Version 4.4.12; TeraRecon, Foster City, California).

\section{Identification of Bleeding Point and Vessel Responsible for Bleeding}

We defined the bleeding point as that point at which the signal of the abnormally extended artery on TOF-MRA overlapped the hypointense area on SWI. We classified the bleeding point into 9 groups, distinguishing between left and right for all sites other than the corpus callosum: 1) thalamus, 2) basal ganglia and internal capsule, 3) periventricular area, 4) corpus callosum, and 5) others, using the minor revised classification of microbleeds (Microbleed Anatomical Rating Scale) ${ }^{15}$ if a bleeding point was detectable (Fig 1). The basal ganglia included the caudate and lentiform nuclei, and the periventricular area was defined as the subependymal and white matter area located within $10 \mathrm{~mm}$ of the wall of the lateral ventricles, except for the thalamus, basal ganglia, and corpus callosum. ${ }^{15}$ In cases of patients who had a history of multiple symptomatic hemorrhages, we referred to patient records and CT images obtained during acute-phase hemorrhage.

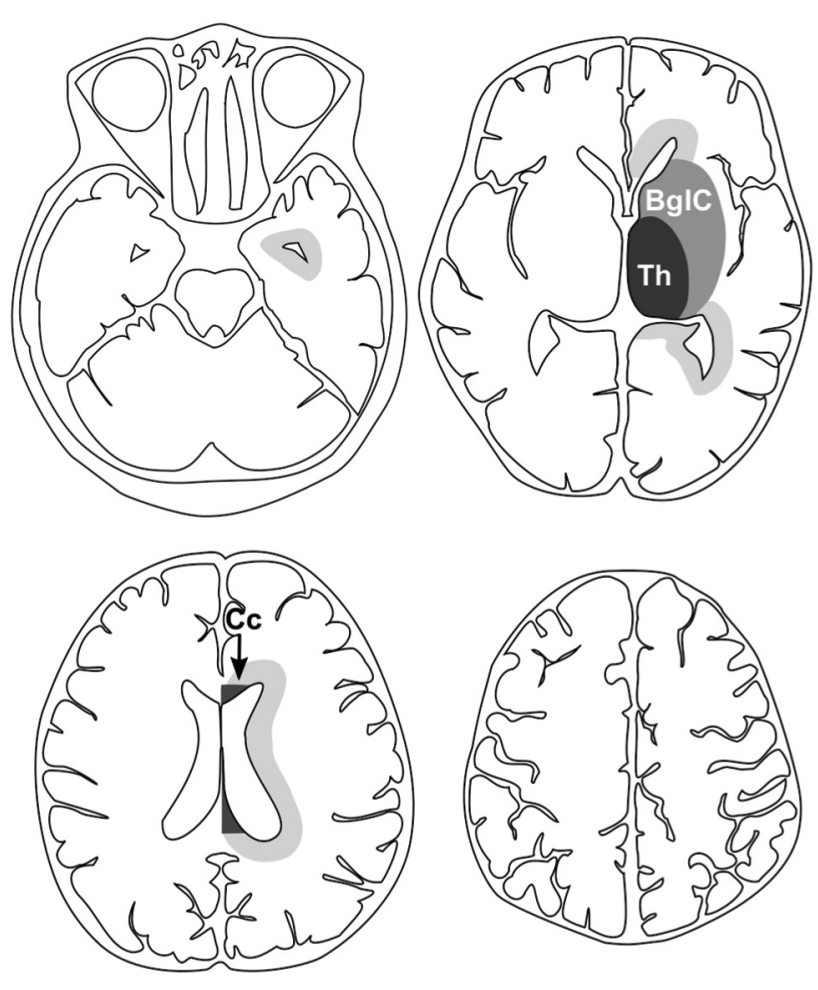

FIG 1. Diagram used for the classification of the site of bleeding. The periventricular area was defined as subependymal tissue and the white matter area located within $10 \mathrm{~mm}$ from the wall of the lateral ventricles except for the thalamus, basal ganglia, and corpus callosum. Th indicates thalamus; BgIC, basal ganglia and internal capsule; Cc, corpus callosum.

Furthermore, we classified the vessel responsible for hemorrhage into 4 types using axial TOF-MRA images and slidingthin-slab maximum-intensity projection images: ${ }^{13}$ 1) thalamic perforator (thalamotuberal artery, thalamoperforating artery or thalamogeniculate artery); 2) lenticulostriate artery; 3) choroidal artery (anterior choroidal artery, medial posterior choroidal artery, or lateral posterior choroidal artery); or 4) other. All bleeding points were drawn in an anatomic diagram showing the type of vessels responsible for bleeding.

Two independent raters (A.M. and T.F.) who were blinded to other clinical information, except for 3 patients with multiple concomitant hemorrhages, assessed whether the bleeding point was detectable and rated the location of bleeding points and responsible vessels. Rater 1 (A.M.) had 12 years of experience in neurosurgery, and rater 2 (T.F.) had 17 years of experience in neurosurgery. Rater 1 rated fusion images twice at an 8 -week interval to determine intrarater reliability. Both raters had participated in a training session involving 5 representative cases in which the bleeding point had been confirmed. In cases of disagreement, we determined bleeding points and vessels as the consensus decision of the 2 raters (A.M. and T.F.) to create the distribution map. After evaluating inter- and intrarater reliability, we confirmed the accuracy of the fusion image in some cases using another workstation (Osirix X DICOM Viewer 10.0; http://www.osirix-viewer.com).

AJNR Am J Neuroradiol 40:1674-80 Oct 2019 www.ajnr.org 


\begin{tabular}{lcccc}
\hline & $\begin{array}{c}\text { Rater } \mathbf{1} \\
(\boldsymbol{n}=\mathbf{4 8})\end{array}$ & $\begin{array}{c}\text { Rater } \mathbf{2} \\
(\boldsymbol{n}=\mathbf{4 8})\end{array}$ & IE (95\% CI) & IA (95\% CI) \\
\hline Presence of responsible vessel & $38(79.2 \%)$ & $35(72.9 \%)$ & $0.83(0.65-1)$ & $0.86(0.68-1)$ \\
Location of bleeding point & $(n=38)$ & $(n=35)$ & $0.92(0.82-1)$ & $0.85(0.72-0.99)$ \\
Thalamus & $3(7.9 \%)$ & $4(11.4 \%)$ & & \\
Basal ganglia and internal capsule & $9(23.7 \%)$ & $7(20 \%)$ & & \\
Periventricular area & $25(65.8 \%)$ & $23(65.7 \%)$ & & \\
Corpus callosum & $1(2.6 \%)$ & $1(2.9 \%)$ & & \\
Other & 0 & 0 & & \\
Origin of responsible vessels & $(n=38)$ & $(n=35)$ & $0.84(0.69-1)$ & $0.90-78-1)$ \\
Lenticulostriate artery & $9(23.7 \%)$ & $10(28.6 \%)$ & & \\
Thalamic perforator & $7(18.4 \%)$ & $4(11.4 \%)$ & & \\
Choroidal artery & $22(57.9 \%)$ & $21(55.3 \%)$ & & \\
Other & 0 & 0 & & \\
\hline
\end{tabular}

Note:-IE indicates interrater; IA, intrarater.

${ }^{a}$ Data are $\kappa$-agreements and correlation coefficients.

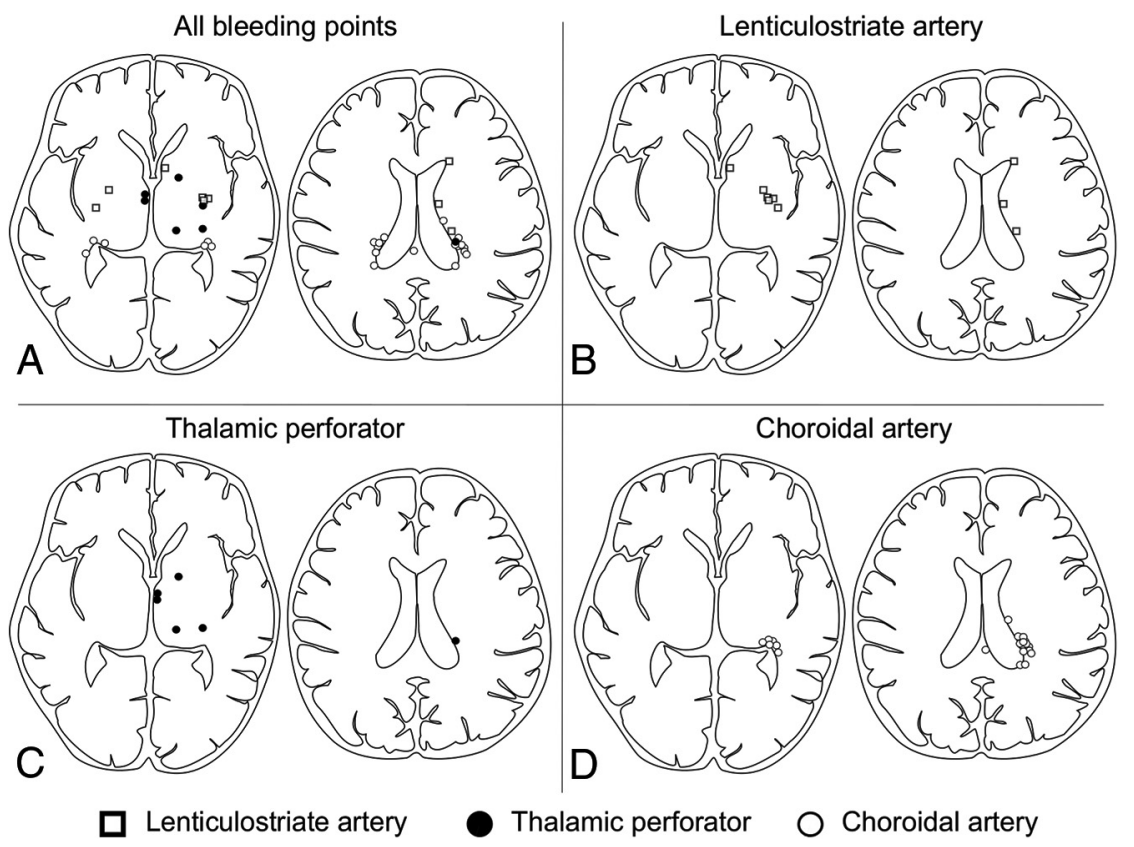

FIG 2. Diagrams show the distribution map of bleeding points and responsible vessels. Squares represent bleeding points derived from lenticulostriate arteries, black circles represent those from thalamic perforators, and white circles represent those from choroidal arteries. A, All bleeding points are drawn in a diagram. $B-D$, Diagrams show bleeding points derived from lenticulostriate arteries, the thalamic perforator, and choroidal artery, respectively. Bleeding points are on the left hemisphere.

\section{Statistical Analysis}

Intra- and interrater agreement for the presence or absence of a bleeding point was calculated using the unweighted $\kappa$ statistic. Classifications of the bleeding point and the vessel responsible for hemorrhage were also calculated using unweighted $\kappa$ statistics. All statistical analyses were conducted using JMP, Version 13 software (SAS Institute, Cary, North Carolina).

\section{RESULTS}

Between January 2009 and April 2018, a total of 55 patients with hemorrhagic Moyamoya disease visited our institution. Thirteen patients were excluded (due to hematoma-evacuation craniotomy, $n=9$; embolization for peripheral anterior choroidal aneurysm, $n=1$; diagnosis of localized primary subarachnoid hemorrhage, $n=1$; patient not having undergone SWI, $n=1$; and patient not being able to undergo construction of fusion images because of errors in imaging processing at scanning, $n=$ $1)$. We investigated the remaining 42 patients, who had experienced 48 hemorrhagic events (3 patients had experienced repeat hemorrhage in different locations).

Seventeen male and 25 female patients with Moyamoya disease were included (median age, 37 years; range, 7-66 years). Of these 42 patients, 8 (19\%) were diagnosed with hypertension and 5 (11.6\%) were diagnosed with dyslipidemia. No patients showed comorbid diabetes mellitus. Twelve patients (28.6\%) had a smoking habit, and 16 patients (38.1\%) had undergone direct or indirect bypass surgery at the time of MR imaging. The median interval from onset to MR imaging was 323.5 days (range, 268683 days).

Intra- and interrater reliabilities for the presence of a bleeding point, site of bleeding, and origin of the responsible vessels are shown in the Table. The detection rate for a bleeding point was $79.2 \%(38 / 48)$ for rater 1 and $72.9 \%$ (35/48) for rater 2 , and interrater reliability was high ( $\kappa=0.83$; 95\% CI, 0.65$1)$. Intrarater reliability of rater 1 for detecting the bleeding point at a 2-month interval was also high $(\kappa=0.86$; 95\% CI, $0.68-1)$. Raters 1 and 2 classified the site of the bleeding point as follows: thalamus, 3 (7.9\%) versus 4 (11.4\%); basal ganglia and internal capsule, $9(23.7 \%)$ versus 7 (20\%); periventricular area, 25 (65.8\%) versus 23 (65.7\%); and corpus callosum, 1 (2.6\%) versus $1(2.9 \%)$, respectively. Interrater and intrarater reliabilities of rater 1 at a 2 -month interval for classification of the site of bleeding were high (interrater: $\kappa=0.92$; 95\% CI, 0.82-1; intrarater: $\kappa=0.85$; $95 \% \mathrm{CI}, 0.72-0.99)$. Raters 1 and 2 determined the origin of the responsible vessel as follows: lenticulostriate artery, 9 (23.7\%) versus 10 (28.6\%); thalamic perforator, 7 (18.4\%) versus 4 (11.4\%); and choroidal artery, 22 (57.9\%) versus 21 (55.3\%), respectively. Interrater and intrarater reliabilities 


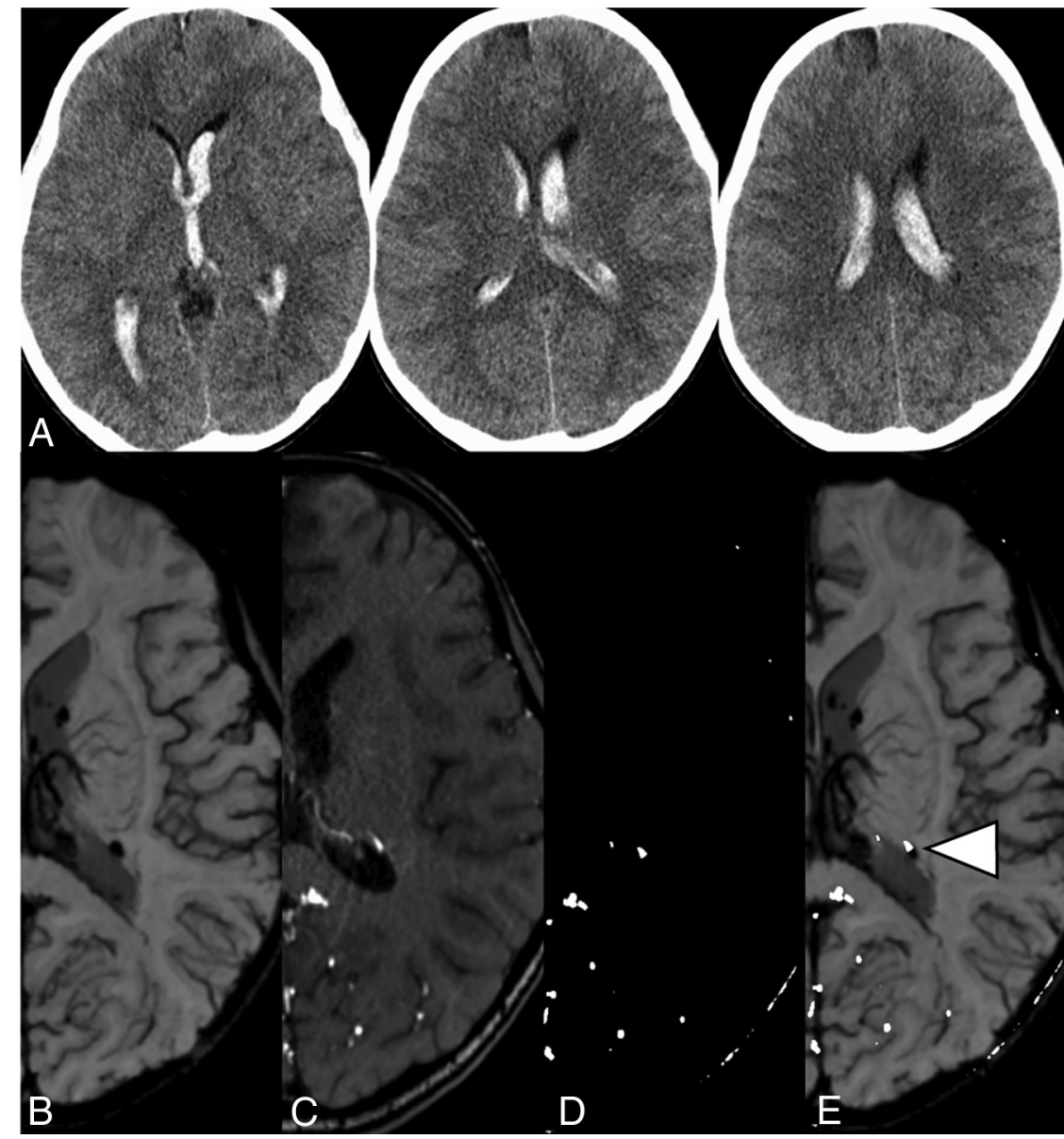

FIG 3. $A, C T$ images from a 7-year-old boy with acute intraventricular hemorrhage. Images of SWI (B) and TOF-MRA $(C)$, contrast-adjusted TOF-MRA $(D)$, and a fusion image of contrast-adjusted TOFMRA and SWI (E) for intraventricular hemorrhage in the same patient (chronic phase). The arrowhead shows the bleeding point at which the signal from the abnormally extended artery on TOFMRA overlaps the hypointense spot on SWI. This point was defined as the bleeding point for the present case.

of rater 1 at a 2 -month interval for the classification of the origin of responsible vessel were both high (interrater: $\kappa=$ 0.84; 95\% CI, 0.69-1; intrarater: $\kappa=0.90$; 95\% CI, $0.78-1$ ). Distributions of bleeding points and vessels responsible for bleeding are depicted in Fig 2.

Sixteen patients had undergone direct or indirect bypass surgery before MR imaging. Twelve patients underwent bypass surgery between the initial hemorrhage and MR imaging, and 4 patients had undergone bypass before initial hemorrhage. In these 16 patients, the detection rate of responsible vessels was $75 \%$ (12 of 16 vessels). After excluding those cases that underwent bypass surgery, the detection rate improved to $84.4 \%$ (27 of 32 events).

Three patients experienced rebleeding twice. One patient developed right putaminal hemorrhage; then a second hemorrhage occurred in the left thalamus. She experienced the third hemorrhage in the right temporal lobe. Another patient developed left putaminal hemorrhage, and the second attack was intraventricular hemorrhage. She experienced the third hemorrhage in the left putamen again. The other patient developed intraventricular hemorrhage, and the second hemorrhage occurred in the right putamen. She presented with intraventricular hemorrhage again as the third attack.

Among the 38 cases in which rater 1 could have detected the bleeding point, 32 sets of angiographic data were obtained. Dilated or extended responsible vessels were confirmed in 30 of the 32 cases on angiograms. Our MR imaging findings corroborated well the results of angiography.

\section{DISCUSSION}

Bleeding points in hemorrhagic Moyamoya disease appeared to be identified with high reproducibility using fusion images of chronicphase SWI and TOF-MRA. SWI images were created using a highresolution gradient-echo method imaged with 3D and corrected flow velocity, and SWI has both high sensitivity for changes in magnetic susceptibility and higher spatial resolution than $\mathrm{T} 2{ }^{\star} \mathrm{WI}{ }^{16,17}$

Detecting the bleeding point in Moyamoya disease using noncontrast or contrast-enhanced CT performed during acute-phase $\mathrm{ICH}$ is sometimes difficult (Figs 3A, 4A, and $5 A$ ), particularly in cases of intraventricular hemorrhage or a large hematoma. On the other hand, chronic-phase SWI and TOF-MRA fusion images demonstrated the bleeding point and responsible artery well (Figs $3 E, 4 E$, and $5 F$ ). SWI for chronicphase hemorrhage offers the advantage of being unaffected by any mass effect of the hematoma (Figs $4 B,-5 C$ ), and evidence of small hematoma in the subependymal region is easily detected by being washed out of intraventricular hematoma (Fig $3 B$ ). In a case of ICH thought of as putaminal hemorrhage in which contrast-enhanced CT could not detect a bleeding point (Fig 5A), angiograms showed dilated lenticulostriate arteries and anterior choroidal artery, but determining the responsible vessel was difficult (Fig 5B). Chronic-phase SWI and TOF-MRA fusion images demonstrated the responsible vessel well; the dilated choroidal artery was thought to have collapsed in the periventricular area in this case (Fig $5 F$ ).

The bleeding point could not be detected on SWI or TOFMRA fusion images in around $20 \%-30 \%$ of cases in the present study. Traces of old hemorrhage were detectable on SWI among each of the 10 cases in which rater 1 could not detect a bleeding point, whereas the signal from the vessels was faint (7 cases) or undetectable (3 cases) on TOF-MRA images. In some cases, an aneurysm had been observed on the dilated perforating artery 


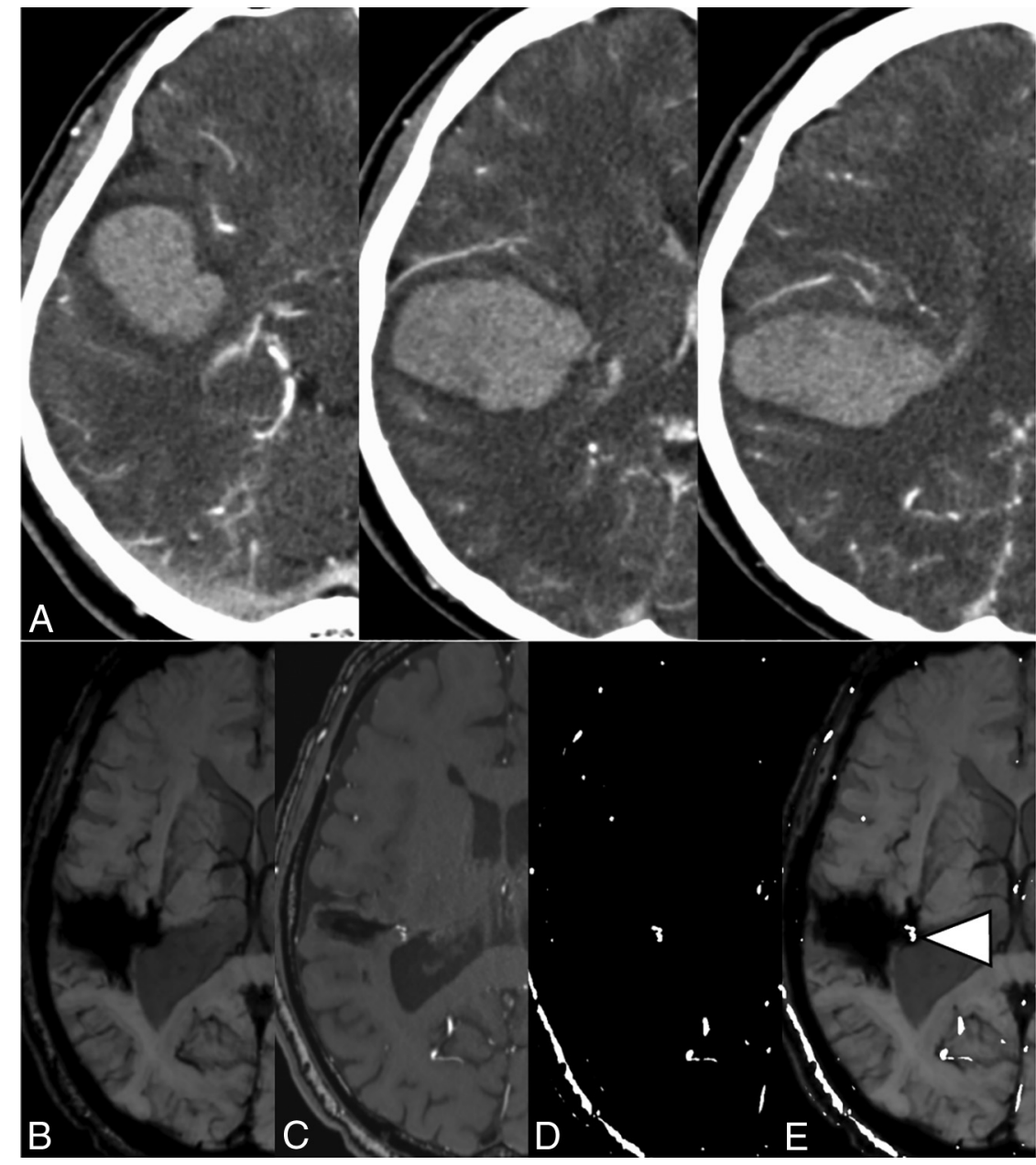

FIG 4. A, Contrast-enhanced CT of a 54-year-old man with acute lobar hemorrhage. Images of SWI $(B)$ and TOF-MRA $(C)$, contrast-adjusted TOF-MRA $(D)$, and a fusion image of contrast-adjusted TOFMRA and SWI (E) in the same patient (chronic phase). The arrowhead shows the signal of an abnormally dilated choroidal artery.

before the hemorrhagic events, but such aneurysms and the dilated perforating artery were not detectable on chronic-phase MRA. In such cases, the responsible vessel might have been blocked during vessel rupture or may have shrunk because the demand for blood flow decreased following damage to the cortex during hemorrhage. In addition, the detection ratio for responsible vessels might have decreased due to the effects of bypass surgery because direct or indirect bypass surgery improves abnormally dilated choroidal arteries and perforators of the posterior communicating artery. ${ }^{3,18}$

Bleeding points were concentrated around the subependymal region, particularly the trigon and posterior parts of the bodies of the lateral ventricles (Fig 2). On the other hand, bleeding points were distributed at the thalamus and putamen, similar to common hypertensive ICH. This distribution pattern corresponds to that described in a past study that investigated bleeding using acute-phase $\mathrm{CT}^{12}$ and also resembles the distribution of microbleeds in Moyamoya disease. ${ }^{10,11}$ Furthermore, the choroidal artery was most frequently detected as the responsible vessel in the periventricular area. Previous studies have shown dilation of the choroidal artery and posterior communicating artery as risk factors for ICH in Moyamoya disease. ${ }^{18-20}$ The development of collateral anastomoses between the choroidal or perforating artery and medullary artery was significantly associated with a hemorrhagic presentation of Moyamoya disease. ${ }^{12,13,21}$ This study suggested that the choroidal artery and perforating artery were at high risk of disruption in the subependymal area in Moyamoya disease. Baltsavias et $\mathrm{al}^{22}$ demonstrated anastomotic connections between the lenticulostriate artery, choroidal artery, or thalamic artery and the medullary artery in the periventricular subependymal white matter using superselective angiography in pediatric patients with Moyamoya disease. That finding corresponds to the concept of periventricular anastomosis as previously reported by Funaki et al. ${ }^{12,13}$ This connection is not present in the normal brain, and thus is speculated to lack the ordinary arterial wall structure at the subependymal area. ${ }^{23-25}$ Hemodynamic stress at this point might lead to collapse of the fragile connection and cause ICH.

Rebleeding attacks were frequent among patients with hemorrhage and Moyamoya disease, and the risk of rebleeding increased with time for 5 years. ${ }^{5}$ This finding suggests that patients with hemorrhage and Moyamoya disease are at risk of rebleeding for a long time. Precise detection and careful observation of the vessel responsible for hemorrhage might help prevent rebleeding. If the responsible vessel enlarges with time, tailored bypass may be able to shrink the vessel and prevent rebleeding. ${ }^{26}$

This study had several limitations that must be considered when interpreting the results. First, although we defined the bleeding point as the point at which the signal from abnormally extended vessel on TOF-MRA overlapped the area of hypointensity on SWI, demonstrating whether this vessel had actually been disrupted is difficult. This issue was mainly because we included only cases that had not undergone hematoma evacuation surgery. Second, gradient-echo imaging such as SWI shows blooming effects proportional to TE and local fields. Overestimation of bleeding size is thus 1 limitation of this study. Quantitative susceptibility imaging has been proposed to eliminate blooming artifacts dependent on gradient echo imaging parameters. Quantitative susceptibility imaging is reported to estimate the size without blooming effects by performing dipole kernel 


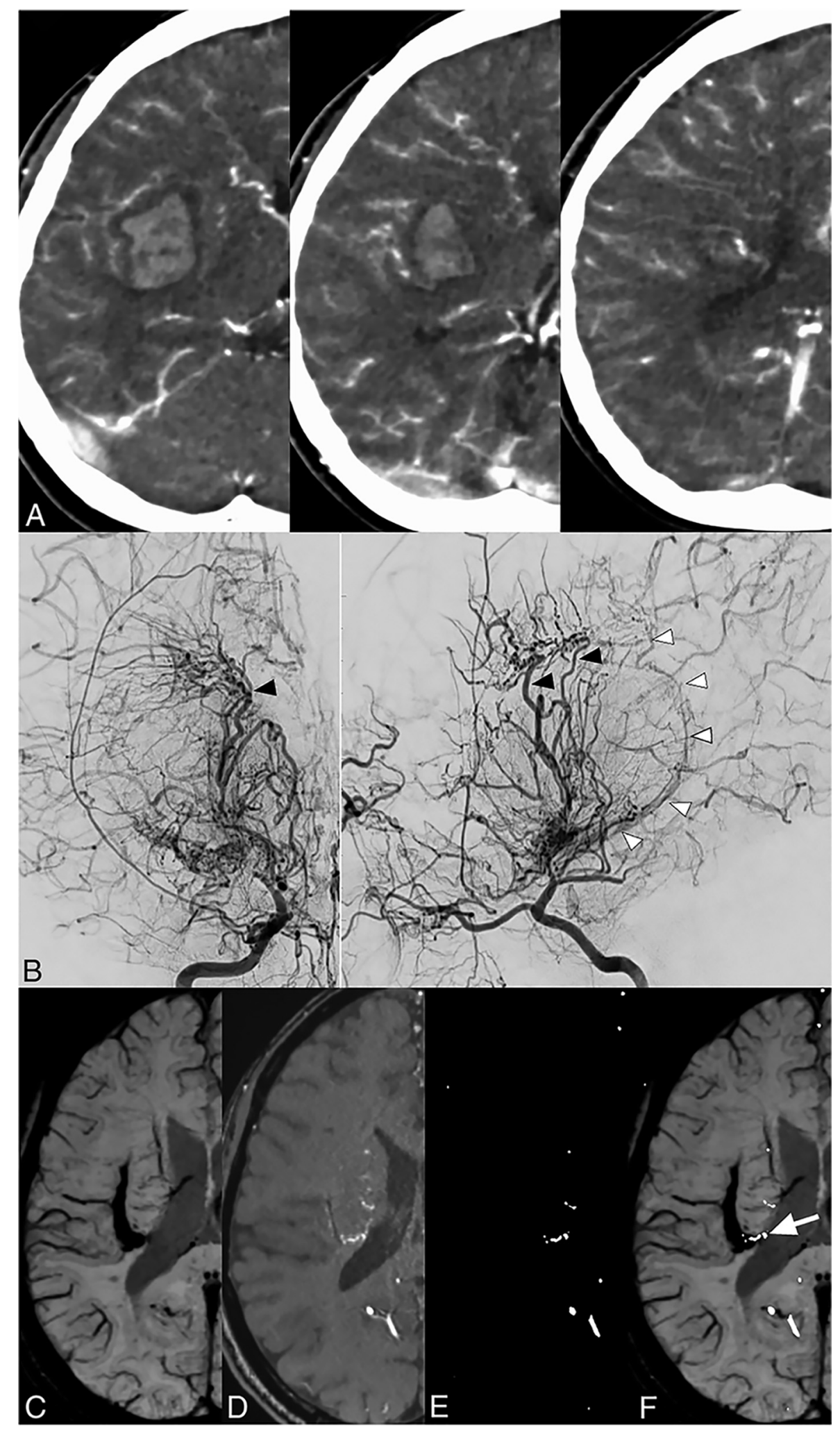

FIG 5. A, Contrast-enhanced CT of a 29-year-old woman with acute putaminal hemorrhage. $B$, Right internal carotid angiogram of the same patient (acute phase) shows abnormal dilation and extension of the lenticulostriate arteries (black arrowheads) and choroidal artery (white arrowheads). Images of SWI (C) and TOF-MRA (D), contrast-adjusted TOF-MRA (E), and fusion image of contrast-adjusted TOF-MRA and SWI ( $)$ in the same patient (chronic phase). The arrow shows the dilated choroidal artery, which might be disrupted in the periventricular area in this case. 
deconvolution. ${ }^{27}$ Third, several cases were seen in which multiple abnormal dilated vessel signs were detected in the low-intensity area on SWI when the hematoma was large. This was the main factor underlying disagreements between the 2 raters.

Chronic-phase SWI and TOF-MRA fusion images are useful to detect bleeding points and the responsible vessel in $\mathrm{ICH}-$ related Moyamoya disease. Further research is needed to establish optimal MR imaging protocols for detecting bleeding points and responsible arteries, and clarification of the features of the responsible artery for ICH might contribute to the prevention of $\mathrm{ICH}$ in Moyamoya disease.

\section{CONCLUSIONS}

Diagnosis using chronic-phase SWI and TOF-MRA fusion images offered good intra- and interrater reliabilities for detecting the bleeding point and responsible vessel. Abnormally dilated perforating and choroidal arteries might offer a key source of ICH in Moyamoya disease. This imaging technique might be clinically useful for accurate identification of the bleeding point, which is often difficult using only the initial CT.

\section{REFERENCES}

1. Han DH, Kwon OK, Byun BJ, et al; Korean Society for Cerebrovascular Disease. A co-operative study: clinical characteristics of 334 Korean patients with Moyamoya disease treated at neurosurgical institutes (1976-1994). Acta Neurochir (Wien) 2000;142: 1263-73 CrossRef Medline

2. Nah HW, Kwon SU, Kang DW, et al. Moyamoya disease-related versus primary intracerebral hemorrhage: location and outcomes are different. Stroke 2012;43:1947-50 CrossRef Medline

3. Jiang $\mathrm{H}$, Yang $\mathrm{H}, \mathrm{Ni} \mathrm{W}$, et al. Long-term outcomes after combined revascularization surgery in adult hemorrhagic Moyamoya disease. World Neurosurg 2018;116:e1032-41 CrossRef Medline

4. Funaki T, Takahashi JC, Houkin $\mathrm{K}$, et al. High rebleeding risk associated with choroidal collateral vessels in hemorrhagic Moyamoya disease: analysis of a nonsurgical cohort in the Japan Adult Moyamoya Trial. J Neurosurg 2018 Feb 1:1-8. [Epub ahead of print] CrossRef Medline

5. Miyamoto S, Yoshimoto T, Hashimoto N, et al. Effects of extracranial-intracranial bypass for patients with hemorrhagic Moyamoya disease: results of the Japan Adult Moyamoya Trial. Stroke 2014; 45:1415-21 CrossRef Medline

6. Liu X, Zhang D, Shuo W, et al. Long term outcome after conservative and surgical treatment of haemorrhagic Moyamoya disease. $J$ Neurol Neurosurg Psychiatry 2013;84:258-65 CrossRef Medline

7. Jang DK, Lee KS, Rha HK, et al. Bypass surgery versus medical treatment for symptomatic Moyamoya disease in adults. J Neurosurg 2017;127:492-502 CrossRef Medline

8. Ge $\mathrm{P}$, Zhang $\mathrm{Q}$, Ye $\mathrm{X}$, et al. Clinical features, surgical treatment, and long-term outcome in children with hemorrhagic Moyamoya disease. J Stroke Cerebrovasc Dis 2018;27:1517-23 CrossRef Medline

9. Takahashi JC, Funaki T, Houkin K, Trial, et al. Significance of the hemorrhagic site for recurrent bleeding: prespecified analysis in the Japan Adult Moyamoya Trial. Stroke 2016;47:37-43 CrossRef Medline
10. Kazumata K, Shinbo D, Ito M, et al. Spatial relationship between cerebral microbleeds, Moyamoya vessels, and hematoma in Moyamoya disease. J Stroke Cerebrovasc Dis 2014;23:1421-28 CrossRef Medline

11. Wenz H, Wenz R, Maros M, et al. Incidence, locations, and longitudinal course of cerebral microbleeds in European Moyamoya. Stroke 2017;48:307-13 CrossRef Medline

12. Funaki T, Takahashi JC, Houkin K, et al. Angiographic features of hemorrhagic Moyamoya disease with high recurrence risk: a supplementary analysis of the Japan Adult Moyamoya Trial. J Neurosurg 2018;128:777-84 CrossRef Medline

13. Funaki T, Takahashi JC, Yoshida K, et al. Periventricular anastomosis in Moyamoya disease: detecting fragile collateral vessels with MR angiography. J Neurosurg 2016;124:1766-72 CrossRef Medline

14. Research Committee on the Pathology Treatment of Spontaneous Occlusion of the Circle of Willis. Health Labour Sciences Research Grant for Research on Measures for Infractable Diseases. Guidelines for diagnosis and treatment of Moyamoya disease (spontaneous occlusion of the circle of Willis). Neurol Med Chir (Tokyo) 2012; 52:245-66 CrossRef Medline

15. Gregoire SM, Chaudhary UJ, Brown MM, et al. The Microbleed Anatomical Rating Scale (MARS): reliability of a tool to map brain microbleeds. Neurology 2009;73:1759-66 CrossRef Medline

16. Haacke EM, Xu Y, Cheng YC, et al. Susceptibility weighted imaging (SWI). Magn Reson Med 2004;52:612-18 CrossRef Medline

17. Sehgal V, Delproposto Z, Haacke EM, et al. Clinical applications of neuroimaging with susceptibility-weighted imaging. J Magn Reson Imaging 2005;22:439-50 CrossRef Medline

18. Morioka M, Hamada JI, Kawano T, et al. Angiographic dilatation and branch extension of the anterior choroidal and posterior communicating arteries are predictors of hemorrhage in adult patients with Moyamoya disease. Stroke 2003;34:90-95 CrossRef Medline

19. Liu W, Zhu S, Wang X, et al. Evaluation of angiographic changes of the anterior choroidal and posterior communicating arteries for predicting cerebrovascular lesions in adult Moyamoya disease. $J$ Clin Neurosci 2011;18:374-78 CrossRef Medline

20. Liu P, Han C, Li DS, et al. Hemorrhagic Moyamoya disease in children: clinical, angiographic features, and long-term surgical outcome. Stroke 2016;47:240-43 CrossRef Medline

21. Fujimura M, Funaki T, Houkin K, et al. Intrinsic development of choroidal and thalamic collaterals in hemorrhagic-onset Moyamoya disease: case-control study of the Japan Adult Moyamoya Trial. J Neurosurg 2018 May 4:1-7. [Epub ahead of print] CrossRef Medline

22. Baltsavias G, Khan N, Valavanis A. The collateral circulation in pediatric Moyamoya disease. Childs Nerv Syst 2015;31:389-98 CrossRef Medline

23. Nelson MD Jr, Gonzalez-Gomez I, Gilles FH. Dyke Award: the search for human telencephalic ventriculofugal arteries. AJNR Am J Neuroradiol 1991;12:215-22 Medline

24. Mayer PL, Kier EL. The controversy of the periventricular white matter circulation: a review of the anatomic literature. AJNR Am J Neuroradiol 1991;12:223-28 Medline

25. Marinkovic S, Gibo H, Filipovic B, et al. Microanatomy of the subependymal arteries of the lateral ventricle. Surg Neurol 2005; 63:451-58 CrossRef Medline

26. Sasagasako T, Funaki T, Tanji M, et al. Intractable medial anastomotic branches from the lenticulostriate artery causing recurrent hemorrhages in Moyamoya disease. World Neurosurg 2019;127:27983 CrossRef Medline

27. Wang S, Lou M, Liu T, et al. Hematoma volume measurement in gradient echo MRI using quantitative susceptibility mapping. Stroke 2013;44:2315-17 CrossRef Medline 